\title{
Instabilities and mixing in stellar radiation zones
}

\author{
Jean-Paul Zahn \\ LUTH, Observatoire de Paris, 92195 Meudon, France \\ email: Jean-Paul.Zahn@obspm.fr
}

\begin{abstract}
The standard model of stellar structure is unable to account for various observational facts, and there is now a large consensus that some 'extra mixing' must occur in the radiation zones. The possible causes for such mixing are briefly reviewed. The most efficient among them is probably the shear-turbulence generated by the differential rotation, which itself results from the transport of angular momentum that can be mediated through the large-scale circulation induced by structural adjustments or by the applied torques (stellar wind, accretion, tides). In solar-type stars this angular momentum transport is ensured mainly by internal gravity waves that are excited at the boundary with convection zones. Another cause of mixing manifests itself in the red giant phase, namely the thermohaline instability due to an inversion of the molecular weight gradient. The implementation of these processes in stellar evolution codes is giving rise to a new generation of stellar models, which are in much better agreement with the observational constraints.
\end{abstract}

Keywords. Instabilities, turbulence, stars: evolution, interiors, rotation

\section{Mixing in stellar radiation zones: the observational evidence}

All along the 20th century, considerable progress has been achieved in modeling stellar interiors, thanks to our great pioneers: Eddington, Chandrasekhar, Bethe, Schwarzschild, Kippenhahn, Iben, and many others. Presently the standard model consists of well-mixed convection zones, whose boundaries are defined by the Schwarzschild criterion, and of stable radiation zones that do not allow for any mixing, except some convective penetration. The most recent improvement was to include the atomic processes that are responsible for the microscopic diffusion of the chemical species, and also for their gravitational settling and radiative acceleration. This standard model proves very successful in predicting the main features of stellar evolution, such as the ascent of the giant branch after the exhaustion of hydrogen in the stellar core. However, some properties of this standard model are in disagreement with a number of observational facts.

Stars of intermediate mass - A-type stars in particular - display anomalies in their surface composition, when they are compared to other, similar stars that are regarded as 'normal'. These anomalies are due to atomic processes, as was shown by Michaud (1970) and his collaborators. Indeed, the radiative acceleration experienced by a given ion depends strongly on its spectral properties, i.e. on its ability of absorbing or diffusing photons, whereas its gravitational settling increases with atomic mass. Depending on which process wins over the other, the considered species appear overabundant or underabundant. But it turns out that these atomic processes are so efficient that they would produce surface anomalies which are much more pronounced than those observed. One consequence, for instance, is that helium would disappear from the surface of A-type stars in about one million years, as was pointed out already by Vauclair et al. (1974) and 
Michaud et al. (1976). Since this is not observed, Vauclair et al. (1978) suggested that some mild turbulence operates near the surface to smooth the composition gradients.

Another sign that some mixing must occur in stellar atmospheres is the striking flatness of the celebrated Spite plateau, with the ${ }^{7} \mathrm{Li}$ abundance in halo stars depending little on effective temperature (Spite \& Spite 1982). If element segregation were alone to operate, the Li depletion would increase with effective temperature, as was recognized by Deliyannis et al. (1990) and Proffitt \& Michaud (1991), and was confirmed recently again by Richard et al. (2002). Therefore one must invoke again some mild mixing occurring below the surface that will enforce the flatness of this plateau.

Furthermore the overabundance, at the surface of massive stars, of chemical elements (such as $\mathrm{He}, \mathrm{N}$ ) that are synthesized in the nuclear core, can only be explained if the radiative interior undergoes some mixing (cf. Meynet \& Maeder 2000). Interestingly, these overabundances seem to be correlated with the rotation velocity of the stars (Herrero et al. 1992, 2000).

A final example is provided by the surface abundances of Li, C and $\mathrm{N}$ of low-mass red giants, after they reach the so-called bump in the luminosity function; these would not appear if there were no extra mixing.

To sum it up, there are many indications that stellar radiation zones do not behave as inert regions, but that they allow for some mild mixing.

\section{How shall one treat that mixing?}

Why should one bother with that mixing? One reason is that it injects hydrogen-rich material in the nuclear core of stars, thus extending their life-time and changing the age estimates based on cluster diagrams. Furthermore, since it modifies the profile of chemical composition within the star, mixing has a strong impact on the later stages of stellar evolution. Also, the matter that is carried away by winds has a non-standard composition, which will orient differently the chemical evolution of the host galaxy.

One way to treat that mixing is what may be considered as the minimalist approach: it consists in introducing a suitably parametrized turbulent diffusivity, and to adjust the parameter(s) to fit the observations. Recently this was done rather successfully by Korn et al. (2006), who showed that a unique parametric prescription for this turbulent diffusivity could explain both the slow rise of the abundance of Fe with evolutionary age in a metal-poor globular cluster, and that of ${ }^{7} \mathrm{Li}$ (followed by its destruction as the convection zone digs deeper). But there is no guarantee that this prescription would work in other circumstances. That is why we prefer the physical approach, namely to strive to implement the physical processes that are likely to cause the mixing.

\section{Mixing processes in the radiation zone of rotating stars}

The main mixing processes that operate in the radiation zone of rotating stars have been identified some time ago (cf. Zahn 1992). These are the large scale circulation which is induced by external torques or structural changes, and the turbulence generated by the shear of differential rotation; their combined action is called rotational mixing.

\subsection{Meridional circulation}

Until recently, the large-scale meridional circulation which occurs in a stellar radiation zone was ascribed to the fact that the isothermal surfaces are distorted by the centrifugal force, with the consequence that the radiative flux is no longer divergence-free (Von Zeipel 1924). In the original treatment (Eddington 1925, Vogt 1925, Sweet 1950), the 
meridional circulation was thus linked to the state of rotation, i.e. the circulation speed and hence the amount mixing scaled proportionally to the square of the angular velocity. The characteristic time was derived by Sweet, and it has since been named the EddingtonSweet time $t_{\mathrm{ES}}$ :

$$
t_{\mathrm{ES}}=t_{\mathrm{KH}} \frac{G M}{\Omega^{2} R^{3}},
$$

with $t_{\mathrm{KH}}=G M^{2} / R L$ being the thermal adjustment time (or Kelvin-Helmholtz time). $R$, $M, L$ designate respectively the radius, mass and luminosity, $\Omega$ the angular velocity and $G$ the gravitational constant. Sweet's result implied that rapidly rotating stars should be well mixed by this circulation, and therefore that they would be prevented from evolving to the giant branch. Since this is not observed, Mestel (1953) invoked the choking effect of the composition gradients that build up due to the nuclear burning.

However, these early studies overlooked the fact that the circulation carries angular momentum: starting from (unknown!) initial conditions, the star undergoes a transient phase which lasts indeed about an Eddington-Sweet time, after which it settles into a quasi-stationary regime where the circulation is governed mainly by the torque applied to the star. For instance, when the star loses angular momentum through a wind, the circulation adjusts such as to transport that momentum to the surface (Zahn 1992). The resulting rotation is then non-uniform, and a baroclinic state sets in, with the temperature varying with latitude along isobars. On the other hand, when the star does not exchange angular momentum, the circulation would die altogether as predicted by Busse (1982), if it had not to compensate a weak turbulent transport down the gradient of angular velocity, because there again the rotation is non-uniform. Thus, in the absence of other processes that will be discussed in $\S 4.2$, it is the loss (or the gain) of angular momentum which drives the circulation and determines its strength, and not the rotation as such, which intervenes only in the transient phases.

A similar circulation is also induced at the boundary of a radiation zone, when the adjacent convection zone is in differential rotation. This occurs in the Sun, where helioseismology has revealed that the rotation rate changes from latitude-dependent in the convection zone, to almost uniform in the radiative interior below, with the transition occurring in a thin boundary layer, the tachocline.

What causes the thinness of the tachocline (it extends less than $5 \%$ in radius) still remains to be elucidated. Indeed, the differential rotation which is imposed by the convection zone should penetrate deep into the radiation zone, through thermal diffusion. Such penetration could be prevented by strongly anisotropic turbulence, as was suggested initially by Spiegel and Zahn (1992). An alternative explanation was put forward by Gough and McIntyre (1998), involving a fossil magnetic field; however we shall see in $\S 4.2 .1$ that this solution is ruled out by recent numerical simulations. But the tachocline spread could be prevented by the cyclic dynamo field, whose penetration is limited by (ohmic) turbulent diffusion - the classical skin effect; that field would act with the shear induced toroidal field to suppress the differential rotation, as was shown by Forgács-Dajka and Petrovay (2002; see also Forgács-Dajka 2004).

\subsection{Shear turbulence caused by differential rotation}

As we have seen, the rotation regime that results from the applied torques is not uniform, and this flow is prone to various instabilities, which generate turbulence and therefore mixing. For a comprehensive review of these instabilities, we refer the reader to Pinsonneault (1997) or Maeder \& Meynet (2000). Here we shall consider only those which apparently play a major role. namely the shear instabilities. 


\subsubsection{Turbulence produced by the vertical shear}

Let us first consider the instability that is produced by the shear in the vertical direction, $\Omega(r)$. This instability is very likely to occur, because the Reynolds number characterizing such shearing flows in stars is extremely high, due to the large sizes involved. Depending on the velocity profile, the instability may be linear, or of finite amplitude. In the absence of stratification, turbulence can be sustained whenever the Reynolds number $R e=w \ell / \nu$ is larger than about $R e_{\mathrm{c}}=40$ (Schatzman et al. 2000). (The Reynolds number $R e$ is expressed here in terms of the velocity $w$ and the size $\ell$ of the largest turbulent eddies, and $\nu$ is the kinematic viscosity.) However the stable entropy stratification in the radiation zone hinders the shear instability: in the absence of thermal dissipation, the instability occurs only if locally

$$
\frac{N^{2}}{\left(\mathrm{~d} V_{\mathrm{h}} / \mathrm{d} z\right)^{2}} \leqslant R i_{\mathrm{c}}
$$

where $V_{\mathrm{h}}$ is the horizontal velocity and $z$ the vertical coordinate. The buoyancy frequency $N$ is given by

$$
N^{2}=\frac{g \delta}{H_{P}}\left[\left(\frac{\partial \ln T}{\partial \ln P}\right)_{\mathrm{ad}}-\frac{d \ln T}{d \ln P}\right],
$$

where $g$ is gravity, $H_{P}$ the pressure scale height and $\delta=-(\partial \ln \rho / \partial \ln T)_{P}$. This condition (3.2) is known as the Richardson criterion; the critical Richardson number $R i_{\mathrm{c}}$ is of order unity, and depends somewhat on the rotational profile.

In a stellar radiation zone, this criterion is modified because the perturbations are no longer adiabatic, due to thermal diffusion. When the radiative diffusivity $K$ exceeds the turbulent diffusivity $D_{\mathrm{v}}=w \ell$, which is generally the case, the instability criterion takes the form (Townsend 1958; Dudis 1974; Zahn 1974; Lignières et al. 1999)

$$
\frac{N^{2}}{\left(\mathrm{~d} V_{\mathrm{h}} / \mathrm{d} z\right)^{2}}\left(\frac{w \ell}{K}\right) \leqslant R i_{\mathrm{c}} \quad \text { when }\left(\frac{w \ell}{K}\right) \leqslant 1 .
$$

From the largest eddies that fulfill this condition, one can estimate the turbulent diffusivity acting in the vertical direction in the radiation zone of a star; it scales as the square of the local shear:

$$
D_{\mathrm{v}}=w \ell=R i_{\mathrm{c}} K \frac{\Omega^{2}}{N^{2}} \sin ^{2} \theta\left(\frac{\mathrm{d} \ln \Omega}{\mathrm{d} \ln r}\right)^{2},
$$

provided that $D_{\mathrm{v}} \geqslant R e_{\mathrm{c}} \nu \approx 40 \nu$.

The instability criterion (3.4) holds as such in regions of uniform composition, where the stability is enforced only by the temperature gradient; when the molecular weight $\mu$ varies with depth, it seems at first sight that one should replace this criterion by the original one, expression (3.2), where now the buoyancy frequency is dominated by the gradient of molecular weight:

$$
N^{2} \rightarrow N_{\mu}^{2}:=\frac{g \varphi}{H_{P}} \frac{\mathrm{d} \ln \mu}{\mathrm{d} \ln P}
$$

with $\varphi=(\partial \ln \rho / \partial \ln \mu)_{P, T}$. As Meynet \& Maeder (1997) pointed out, this very severe condition would prevent any mixing in early-type main-sequence stars, contrary to what is observed. We shall see next how that stabilizing action of $\mu$-gradients can be overcome.

\subsubsection{Turbulence produced by the horizontal shear}

Likewise, the horizontal shear $\Omega(\theta)$ will also generate turbulence, probably through a finite-amplitude instability, because most plausible rotation profiles in stars are linearly 
stable. What type of turbulence then occurs is still a matter of debate. Its vertical component will be constrained by the stratification and therefore it is likely that this turbulence will be highly anisotropic, with much stronger transport in the horizontal than in the vertical direction, i.e. $D_{\mathrm{h}} \gg D_{\mathrm{v}}$.

The postulated anisotropy seems plausible, given the strong stratification in stellar radiation zones and the fact that no restoring force opposes the horizontal displacements; such turbulence was conjectured already in Zahn (1975). We shall further assume that this turbulence acts to suppress its cause, namely the differential rotation in latitude. This will thus lead to a 'shellular' rotation state, where the angular velocity depends little on latitude, and where we can assume that $\Omega \sim \Omega(r)$.

An interesting property of such anisotropic turbulence is that it interferes with the advective transport due to the meridional circulation, and turns it into a vertical diffusion, as it was pointed out by Chaboyer \& Zahn (1992). To first approximation, the vertical component of the circulation velocity is given by $u_{r}(r, \theta)=U_{2}(r) P_{2}(\cos \theta)$, where $P_{2}$ is the Legendre polynomial of degree 2 ; then the resulting effective diffusivity is

$$
D_{\text {eff }}=\frac{1}{30} \frac{\left(r U_{2}\right)^{2}}{D_{\mathrm{h}}}
$$

provided that $r U_{2} \geqslant D_{\mathrm{h}}$. Unfortunately, a reliable prescription for that horizontal diffusivity $D_{\mathrm{h}}$ is still lacking, in spite of recent developments. Maeder (2003) derived a new expression from energy considerations, whereas Mathis et al. (2004) adapted the prescription drawn from laboratory experiments by Richard \& Zahn (1999); the two recipes give similar results (which does not mean that they are valid).

Another property of such anisotropic turbulence is that, by smoothing out chemical inhomogeneities on level surfaces, it reduces the stabilizing effect of the vertical $\mu$-gradient. The Richardson criterion for the vertical shear instability then involves also the horizontal diffusivity $D_{h}$, and the vertical component of the turbulent viscosity can be derived as before (Talon \& Zahn 1997):

$$
D_{\mathrm{v}}=R i_{\mathrm{c}}\left[\frac{N_{T}^{2}}{K+D_{\mathrm{h}}}+\frac{N_{\mu}^{2}}{D_{\mathrm{h}}}\right]^{-1} \sin ^{2} \theta\left(\frac{\mathrm{d} \Omega}{\mathrm{d} \ln r}\right)^{2},
$$

where $N_{T}^{2}$ stands for the thermal part of the buoyancy frequency given by expression (3.3). Thanks to this horizontal erosion, the $\mu$-gradients are much less effective in hindering the mixing.

\section{Rotational mixing}

The two transport processes that have just been discussed (meridional circulation and shear-induced turbulence) are both linked with the (differential) rotation. Therefore, when modeling the evolution of a star including these mixing processes, it is necessary to calculate also the secular evolution of its rotation rate $\Omega(r, \theta)$. The problem is simple to handle, if one deals just with laminar flows. However, as already mentioned, the differential rotation which is generated by this advection generates turbulence, which in turn transports angular momentum. In addition, torques may be applied to the star, either externally due to mass loss or accretion, or to tides exerted by a companion or an accretion disk, or else internally, due to magnetic stresses and to internal gravity waves, as we shall see next. To take all these effects into account, one has thus to solve the 
complete transport equation for the angular momentum:

$$
\frac{\partial}{\partial t}\left[\rho r^{2} \sin ^{2} \theta \Omega\right]+\nabla \cdot\left[\rho \mathbf{u} r^{2} \sin ^{2} \theta \Omega\right]=\text { applied torques }
$$

$\mathbf{u}$ being the circulation velocity.

The problem may be significantly simplified if the horizontal shear is nearly suppressed by the anisotropic turbulence mentioned above. To lowest order $\Omega$ is then a function of $r$ only, and all perturbations from the non-rotating state separate in $r$ and colatitude $\theta$, as illustrated here for the vertical component of the meridional velocity: $u_{r}(r, \theta)=U_{2}(r) P_{2}(\cos \theta)$. For a detailed account of how this modelization is implemented, we refer to Zahn (1992), Maeder \& Zahn (1998) and Mathis \& Zahn (2004).

\subsection{Rotational mixing of type $I$}

We first examine the simplest case, that we call 'rotational mixing of type I', where the angular momentum is transported by the same processes that are responsible for the mixing, namely the meridional circulation and the turbulent diffusion. The angular velocity $\Omega(r)$ then obeys the following transport equation, obtained by averaging equation (4.1) over $\theta$ :

$$
\frac{\partial}{\partial t}\left[\rho r^{2} \Omega\right]=\frac{1}{5 r^{2}} \frac{\partial}{\partial r}\left[\rho U_{2} r^{2} \Omega\right]+\frac{1}{r^{2}} \frac{\partial}{\partial r}\left[\rho \nu_{\mathrm{v}} r^{4} \frac{\partial \Omega}{\partial r}\right],
$$

with $\nu_{\mathrm{v}} \approx D_{\mathrm{v}}$ given by equation (3.8). In spite of the fact that this equation is only onedimensional, it captures the advective character of the angular momentum transport by the meridional circulation: depending on the sign of $U_{2}$, i.e. on the sense of the circulation in each hemisphere, angular momentum may be transported up the gradient of $\Omega$, which is never the case when the effect of meridional circulation is modeled just as a diffusive process, as it is often done (cf. Pinsonneault et al. 1989 or Chaboyer et al. 1995).

We stated already that the circulation is governed mainly by the applied torques. When there is no or little loss of angular momentum from the star, the circulation settles into a regime of differential rotation where a weak inward flux of angular momentum compensates the turbulent diffusion directed outwards, down the gradient of $\Omega(r)$. On the other hand, when the star loses angular momentum (for instance through a wind), the circulation adjusts itself such as to transport the required amount of angular momentum towards the surface (Zahn 1992).

Massive main-sequence stars belong to the first category, and the implementation of rotational mixing has been quite successful in improving their models (Maeder \& Meynet 2000). The theoretical isochrones fit well the observed ones, even without introducing convective overshoot from the core, and such rotational mixing accounts well for the observed enhancement of $\mathrm{He}$ and $\mathrm{N}$ at the surface of early-type stars. This was first illustrated with the evolution of a $9 M_{\odot}$ star by Talon et al. (1997), and then generalized to all massive stars by Meynet and Maeder (2000). Combined with a suitable prescription for the mass loss, this type of mixing also yields the observed proportion of blue and red giants. Finally, such mixing accounts perfectly for the destruction of $\mathrm{Li}$ on the blue side of the Li gap, as was shown by Charbonnel and Talon (1999); in their calculations they took the same value for the adjustable parameter characterizing the anisotropic turbulence than that which was used to model the $9 M_{\odot}$ star by Talon et al. (1997).

The second case applies to solar-type stars, whose modeling has been much less successful, until very recently. In those stars, which lose most of their angular momentum in their youth through a magnetized wind (Schatzman 1962), the meridional circulation adjusts so as to carry the required angular momentum towards the surface, at least in the absence of other processes (Zahn 1992). One would then expect that the amount of 
mixing, and hence the depletion of light elements, be proportional to the loss of angular momentum. This would have as consequence that tidally-locked binaries would destroy less Li than single stars, since the angular momentum carried away by their winds would not be drawn from their internal rotation, but from their orbital motion; hence there would be no need to transport angular momentum inside such stars, and their Li would be preserved (Zahn 1994). However, there is no sign that tidally-locked binaries are less Li-depleted than single stars, as was shown by Balachandran (2002); hence one must conclude that the transport of matter is not correlated with the transport of angular momentum.

Another hint is provided by the fact that lithium is seriously depleted in the Sun, and that the somewhat less fragile beryllium is not, as was shown by Balachandran and Bell (1997). This contrasted behavior may be explained if the mixing occurs only in the vicinity of the convection zone, i.e. in the tachocline. As a matter of fact, the meridional circulation which is induced in that layer by the differential rotation of the convection zone (see section 3.1), can produce such localized mixing, as was shown by Brun et al. (1999).

But the most severe observational test is that models built according to equation (4.2), i.e. including only turbulence and meridional circulation, conserve a rapidly spinning core (Pinsonneault et al. 1989; Matias \& Zahn 1998), which is ruled out by helioseismology. We must therefore conclude that another, more powerful process is responsible for the transport of angular momentum in solar-type stars; it is that process which then shapes the rotation profile and therefore, though indirectly, determines the extent of mixing.

\subsection{Rotational mixing of type $I I$}

In what we call rotational mixing of type II, the chemical elements are still transported by the meridional circulation and the turbulence caused by differential rotation, but the angular momentum is carried by another process, which is powerful enough to establish an almost uniform rotation profile in the solar radiative interior. Two candidates have been proposed for this extra transport: magnetic torquing and internal gravity waves.

\subsubsection{Magnetic field}

Magnetic fields, because they are almost 'frozen' in the highly conducting stellar material, are very powerful in reducing differential motions. This was already pointed out by Mestel (1953), who claimed that a fossil magnetic field of moderate strength would render stellar rotation nearly uniform. More precisely, in presence of an axisymmetric poloidal field, the angular velocity tends to become uniform along the field lines, a property referred to as Ferraro's law (1937). Thus if the poloidal field lines lie entirely in the radiation zone of a star, they impose there uniform rotation.

The situation changes however when the poloidal field connects with a differentially rotating convection zone, such as that in the Sun. Then this differential rotation is transmitted along the field lines, and the result is a non uniformly rotating radiation zone, as illustrated by the calculations made by Charbonneau and MacGregor (1993). Can this fate be prevented, for instance if the field is advected by a suitable flow?

Gough and McIntyre (1998) sketched a solution to this problem in which the ohmic diffusion of the fossil field into the solar convection zone was prevented by the down-flow associated with the thermally driven circulation in the tachocline. In turn, that field was invoked to oppose the penetration of the tachocline into the deeper interior. The authors were aware of course that the circulation had also up-flows, which would advect the field into the convection zone, but if these up-flows cover only a small fraction of the domain, the field would imprint a small amount of differential rotation in the radiation zone. 
Clearly, more detailed calculations were required to settle the question. The first of these were performed by Garaud (2002), who built a set of stationary solutions for various parameters. These solutions already hinted that the field lines would penetrate into the convection zone over a broad band of high latitudes, and therefore imprint a substantial amount of differential rotation in the radiation zone. This behavior has been confirmed by Brun and Zahn (2006) through 3-dimensional time-dependent calculations: they found that even a deeply buried poloidal magnetic field will eventually connect with the convection zone, and induce differential rotation below. We are thus led to conclude that, in the Sun at least, it is not the magnetic field that is responsible for the uniform rotation of the radiative interior.

However in other stars, such as magnetic A-type stars, fossil fields presumably play a much more important role, as was recently shown by Braithwaite and Spruit (2004): they certainly interfere with the circulation to modify the mixing in the radiation zone (Mathis \& Zahn 2005). Moreover, certain field configurations are unstable, and they could produce MHD turbulence, and possibly mixing, before they relax into a stable state.

This is now being explored intensively through numerical simulations; the first indication we have gleaned from them (Zahn et al. 2007) is that this MHD turbulence behaves rather as a superposition of Alfvén waves, and that it does not produce any mixing.

\subsubsection{Internal gravity waves}

Since magnetic fields seem unable to enforce uniform rotation in the radiative interior of solar-type stars, we must turn to the other possible mechanism, namely the transport of angular momentum by the internal gravity waves emitted by the turbulent motions at the base of the convection zone. The importance of that process was already anticipated by Press (1981) and Schatzman (1993).

The restoring force operating on gravity waves is the buoyancy force: therefore they travel only in stably stratified regions, i.e. in radiation zones. Presumably a whole spectrum of such waves is emitted at the base of the convection zone of late-type stars; they penetrate into the radiation zone, transporting angular momentum which they deposit wherever they are dissipated through radiative damping. It is by shaping the rotation profile that they indirectly participate in the mixing of chemicals.

Let us first examine the behavior of those waves which are of short wavelength, and are dissipated close to the convection zone. Prograde waves carry positive angular momentum, retrograde waves negative angular momentum. When they travel in a medium which is rotating faster then the region where they have been emitted, their frequency is Doppler-shifted, leading to higher dissipation for the prograde waves than for the retrograde waves. For this reason the angular velocity tends to increase where it was already high, and its slope with depth steepens until the shear becomes unstable. That turbulent layer then merges with the convection zone. But in the meanwhile the retrograde waves have deposited negative angular momentum somewhat further down, thus building there another shear layer of opposite direction, which now takes the place of the former one. And the cycle repeats. A similar phenomenon is observed in the Earth atmosphere, where it is called the quasi-biennial oscillation (cf. McIntyre 1994).

The question then arises how this very thin shear layer, located just below the convection zone, affects the waves of longer period and wavelength, which are much less damped, and are therefore able to reach the core of the star. If there is no slope in angular velocity, the shear layer oscillation is perfectly symmetrical in time, and its effect is the same on the prograde and on the retrograde waves. But if $\Omega$ increases even slightly with depth in that layer, the prograde waves will be more dissipated, which allows the retrograde waves to extract angular momentum from the deep interior. This scenario 
has been tested through numerical simulations performed by Talon et al. (2002), using a rather crude approach where the Coriolis force was neglected and where an arbitrary turbulent viscosity was imposed. Recently it has been confirmed through more detailed and more realistic calculations performed by Talon and Charbonnel; beside the internal gravity waves, they include also the meridional circulation and the shear induced turbulence (Talon \& Charbonnel 2003, 2004, 2005). Their calculations are one-dimensional, with the flux of angular momentum averaged over level surfaces, which are assumed to rotate uniformly due to the anisotropic shear-induced turbulence discussed above.

The result is spectacular: internal gravity waves succeed in achieving nearly uniform rotation in solar-type stars at the solar age (see also Charbonnel \& Talon 2005). Furthermore their models predict the observed $\mathrm{Li}$ abundances as a function of age, which is a crucial test to validate the mixing processes. They explain the Spite plateau for population II stars, and the Li dip in galactic clusters. For the first time, we now have a coherent and consistent picture of internal mixing in all main sequence stars!

\section{Thermohaline mixing}

Numerous spectroscopic observations provide compelling evidence for a non-canonical mixing process that modifies the surface abundances of $\mathrm{Li}, \mathrm{C}$ and $\mathrm{N}$ of low-mass red giants when they reach the bump in the luminosity function. These abundance patterns cannot explained by the rotational mixing described above (see Charbonnel \& Palacios 2004). Based on numerical simulations, Eggleton et al. (2006) proposed that a molecular weight inversion created by the ${ }^{3} \mathrm{He}\left({ }^{3} \mathrm{He}, 2 \mathrm{p}\right){ }^{4} \mathrm{He}$ reaction may be at the origin of this mixing, and they ascribed it to the Rayleigh-Taylor instability.

In fact, while the inverse $\mu$-gradient gradually builds up below the convective envelope, the first instability that appears is the thermohaline instability, as it was shown by Charbonnel and Zahn (2007). This instability is well known in oceanography, where it arises when salty warm water lies above cold fresh water (Stern 1960). In the laboratory, the instability takes the form of 'salt fingers'; since heat diffuses faster than salt, these fingers sink because they grow increasingly heavier than their environment, until they become turbulent and dissolve. In stellar interiors, the role of salt is played by a heavier species, such as helium in a hydrogen-rich medium.

In a star, this instability occurs in a stable stratification that satisfies the Ledoux criterion for convective stability, but where the molecular weight decreases with depth:

$$
N_{T}^{2}+N_{\mu}^{2}>0, \quad N_{\mu}^{2}<0 .
$$

(The buyoancy frequencies $N_{T}^{2}$ and $N_{\mu}^{2}$ have been defined above in $\S 3.2 .1$.)

In its fully developed regime, the thermohaline instability manifests itself as a complex phenomenon, displaying fingers, 'staircases', collective instabilities, etc. Numerical simulations reveal that its properties strongly depend on the governing parameters, and on the applied boundary conditions. As yet, there is no simple way to describe the mixing achieved by that instability in stars. Lacking of something better, one can follow Ulrich (1972), who assimilated this mixing to a diffusive process, and was the first to derive a prescription for the turbulent diffusivity; for a general equation of state, it is

$$
D_{t}=C_{t} K \frac{-N_{\mu}^{2}}{N_{T}^{2}+N_{\mu}^{2}} \quad \text { for } N_{\mu}^{2}<0,
$$

$K$ being the thermal diffusivity. Ulrich's non-dimensional coefficient $C_{t}$ involves the aspect ratio $\alpha$ (length/width) of the fingers: $C_{t}=8 \pi^{2} \alpha^{2} / 3$; for the value he advocates, $\alpha=5$, this coefficient is rather large: $C_{t}=658$. 


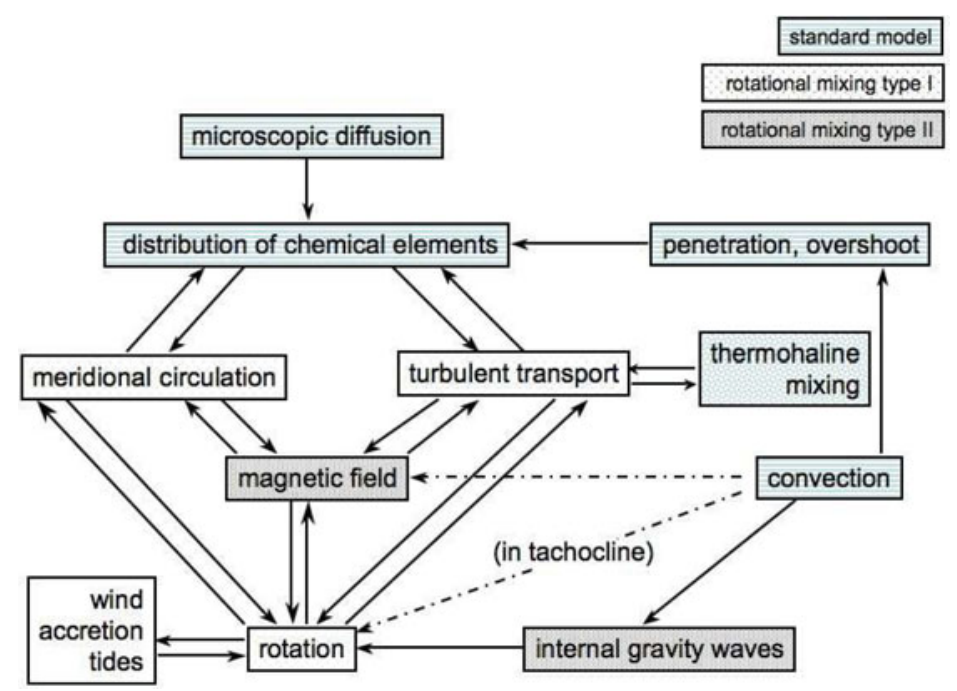

Figure 1. Mixing processes in a stellar radiation zone: a highly non-linear problem, with multiple interactions. In the standard model, the only mixing processes taken into account are convective penetration and microscopic diffusion. Rotational mixing involves the meridional circulation induced by external torques or structural changes and the shear turbulence due to differential rotation; in rotational mixing of type I, angular momentum is carried exclusively by the same processes. In rotational mixing of type II, the angular momentum is transported either by magnetic stresses or by internal gravity waves. (Adapted from Mathis \& Zahn 2005, courtesy $\mathrm{A} \& \mathrm{~A})$.

When applying this prescription to a evolutionary sequence, the models predict the abundance ratios $\left[{ }^{7} \mathrm{Li} / \mathrm{H}\right],\left[{ }^{12} \mathrm{C} /{ }^{13} \mathrm{C}\right],[\mathrm{C} / \mathrm{Fe}]$ and $[\mathrm{N} / \mathrm{Fe}]$ that are in excellent agreement with the observed values, as it was shown by Charbonnel and Zahn (2007).

Elsewhere in this volume, S. Vauclair describes another occurrence of thermohaline mixing, when a star accretes heavy material, such as telluric planets (see Vauclair 2004).

\section{Conclusion}

To conclude, we are entering a new era of modeling stellar interiors, where rotation will be explicitly taken into account, and where the transport processes discussed above will be implemented in the models. This is not an easy task, because it involves intricate couplings, as illustrated by the flow chart displayed in Fig. 1. The (differential) rotation is responsible for the meridional circulation and the shear-induced turbulence, which transport angular momentum and react back on the rotation profile. These mechanisms modify the distribution of chemical elements, which itself interferes with them. A magnetic field modifies the rotation and therefore also the circulation and the turbulent transport; it may become unstable and will then contribute to the mixing. Internal gravity waves emitted at the edge of convection zones will transport angular momentum, and modify the rotation profile. Furthermore some mixing occurs in the vicinity of convection zones, due to convective penetration, and to the circulation induced in the tachoclines through the differential rotation imposed by the adjacent convection zones. Finally, thermohaline mixing occurs in stably stratified regions, when the molecular weight increases with height. 
Most pieces of this scheme are now based on sound physics and robust prescriptions, but some weaknesses remain to be remedied.

Above all, we need to improve the description of the turbulent transport due to shear instabilities, in particular that operating in the horizontal direction. Also, the way we handle convective penetration and the generation of internal gravity waves is far from satisfactory - but the same could be said about our present treatment of the convection zones. We must clarify whether the gravity waves are able to diffuse chemicals, beside transporting angular momentum, as was argued by Schatzman (1993). We still don't know for sure which physical process prevents the spread of the solar tachocline. We have to introduce magnetic fields in our models, at least where we think that they could play a role. Finally, we are in great need of a reliable prescription for the thermohaline mixing. In all these subjects, there is much to be expected from the high-resolution numerical simulations that are now undertaken by several teams.

In the meanwhile, the transport processes discussed above have, as they stand, already been introduced in several stellar evolution codes, in collaborative efforts such as described by Decressin et al. (2008). Their treatment will continue to greatly benefit from observational constraints, in particular from those we anticipate from asteroseismology. It is clear that in not so distant future those non-standard mixing processes will be an integrant part of the standard model.

\section{References}

Balachandran, S. C. 2002, Highlights of Astronomy 12, 276

Balachandran, S. C. \& Bell, R. A. 1997, BAAS, 29, 1325

Braithwaite, J. \& Spruit, H. C. 2004, Nature 431, 819

Brun, A. S., Turck-Chièze, S., \& Zahn, J.-P. 1999, ApJ 525, 1032

Brun, A. S. \& Zahn, J.-P. 2006, A\&A 457, 665

Busse, F. H. 1982, ApJ 259, 759

Chaboyer, B., Demarque, P., \& Pinsonneault, M. H. 1995, ApJ 441, 865

Chaboyer, B. \& Zahn, J.-P. 1992, A\&A 253, 173

Charbonneau, P. \& MacGregor, K. B. 1993, ApJ 417, 762

Charbonnel, C. \& Palacios, A. 2004, IAU Symp. 215, 440

Charbonnel, C. \& Talon, S. 1999, A\&A 351, 635

Charbonnel, C. \& Talon, S. 2005, Science 309, 2189

Charbonnel, C. \& Zahn, J.-P. 2007, A\&A 467, L15

Decressin, T., Mathis, S., Palacios, A., Siess, L., Talon, S., Charbonnel, C., \& Zahn, J.-P. 2008, A\&A (submitted)

Deliyannis, C, Demarque, P., \& Kawaler, S. 1990, ApJS 73, 21

Dudis, J. J. 1974, J. Fluid Mech. 64, 65

Eddington, A. S. 1926, Observatory 48,73

Eggleton, P. P., Dearborn, D. S. P., \& Lattanzio, J. C. 2006, Science 314, 1580

Ferraro, V. C. A. 1937, MNRAS 97, 458

Forgács-Dajka, E. 2004, A\&A 413, 1143

Forgács-Dajka, E. \& Petrovay, K. 2002, A\&A 389, 629

Garaud, P. 2002, MNRAS 329, 1

Gough, D. O. \& McIntyre, M. E. 1998, Nat 394, 755

Herrero, A., Kudritski, R. P., Vilchez, J. M. et al. 1992, A\&A 261, 209

Herrero, A., Puls, J., \& Villamariz, L. R. 2000, A\&A 354, 193

Korn, A. J., Grundahl, F., Rochard, O., Barklem, P. S., Mashonkina, L., Collet, R., Piskunov, N., \& Gustavsson, B. 2006, Nat 442, 657 
Lignières, F., Califano, F., \& Mangeney, A. 1999, A\&A 349, 1027

Maeder, A. 2003, A\&A 399, 263

Maeder, A. \& Meynet, G. 2000, ARA\&A 38, 143

Maeder, A. \& Zahn, J.-P. 1998, A\&A 334, 1000

Mathis, S., Palacios, A., \& Zahn, J.-P. 2004, A\&A 425, 243

Mathis, S. \& Zahn, J.-P. 2004, A\&A 425, 229

Mathis, S. \& Zahn, J.-P. 2005, A\&A 440, 653

Matias, J. \& Zahn, J.-P. 1998, Sounding Solar and Stellar Interiors, IAU Symp. 181, (ed. Provost, J. \& Schmider, F.-X.) poster vol. p. 103

McIntyre, M. E. 1994, The Solar Engine and its Influence on the Terrestrial Atmosphere and Climate (NATO ASI Subseries I, Global Environmental Change) 25, 293 (Cambridge Univ. Press), p. 557

Mestel, L. 1953, MNRAS 113, 716

Meynet, G. \& Maeder, A. 1997, A\&A 321, 465

Meynet, G. \& Maeder, A. 2000, A\&A 361, 101

Michaud, G. 1970, ApJ 160, 641

Michaud, G., Charland, Y., Vauclair, S., \& Vauclair, G. 1976, ApJ 210, 447

Pinsonneault, M. 1997, ARA\&A 35, 557

Pinsonneault, M., Kawaler, S. D., Sofia, S., \& Demarque, P. 1989, ApJ 338, 424

Press, W. H. 1981, ApJ 245, 286

Proffitt, C. R. \& Michaud, G. 1991, ApJ 371, 584

Richard, D. \& Zahn, J.-P. 1999, A\&A 347, 734

Richard, O., Michaud, G., \& Richer, J. 2002, ApJ 568, 979

Schatzman, E. 1962, Ann. Ap 25, 18

Schatzman, E. 1993, A\&A 279, 431

Schatzman, E., Zahn, J.-P., \& Morel, P. 2000, A\&A 364, 876

Spiegel, E. A. \& Zahn, J.-P. 1992, A\&A 265, 106

Spite, F. \& Spite, M. 1922, A\&A 115, 357

Stern, M. E., 1960, Tellus, 12, 172

Sweet, P. A. 1950, MNRAS 110, 548

Talon, S. \& Charbonnel, C. 2003, A\&A 405, 1025

Talon, S. \& Charbonnel, C. 2004, A\&A 418, 1051

Talon, S. \& Charbonnel, C. 2005, A\&A 440, 981

Talon, S., Kumar, P., \& Zahn, J.-P. 2002, ApJ 574L, 175

Talon, S. \& Zahn, J.-P. 1997, A\&A 317, 749

Talon, S., Zahn, J.-P., Maeder, A., \& Meynet, G. 1997, A\&A 322, 209

Théado, S. \& Vauclair, S. 2001, A\&A 375, 70

Townsend, A. A. 1958, J. Fluid Mech. 4, 361

Ulrich, R. K., 1972, ApJ, 172, 165

Vauclair, G., Vauclair, S., \& Michaud, G. 1978, ApJ 223, 920

Vauclair, G., Vauclair, S., \& Pamjatnikh, A. 1974, A\&A 31, 63

Vauclair, S. 1999, A\&A 351, 973

Vauclair, S. 2004, ApJ 605, 874

Vitense, E. 1953, Z. Astrophys. 32, 135

Vogt, H. 1925, Astron. Nachr. 223, 229

Von Zeipel, H. 1924, MNRAS 84, 665

Zahn, J.-P. 1974, Stellar Instability and Evolution, IAU Symp. 59, p. 185

Zahn, J.-P. 1975, Mém. Soc. Roy. Sci. Liège 6 série, 8, 31

Zahn, J.-P. 1991, A\&A 252, 179

Zahn, J.-P. 1992, A\&A 265, 115

Zahn, J.-P. 1994, A\&A 288, 829

Zahn, J.-P., Brun, A. S., \& Mathis, S. 2007, A\&A 475, 145 


\section{Discussion}

CAnuto: Salt-Finger existence is heavily dependent on the strength of the shear present whatever its origin - Lab data on SF do not include shear av therefore they must be used with care when applied to the real world - A theory of SF+Turbulence has recently been proposed.

ZAHN: I agree that shear will play a role, and we know that there is shear because of differential rotation. 